\title{
MIEDOS EN ADULTOS: ESTRUCTURA, EVOLUCIÓN Y CORRELATOS
}

\author{
Vicente Pelechano
}

Universidad de La Laguna (Tenerife)

\section{RESUMEN}

Se presentan resultados de validación de un inventario de miedos para adultos. La prueba ha sido cumplimentada de forma individual por 576 adultos (17a 65 años) de ambos géneros y además se han cumplimentado otras pruebas de personalidad (cinco grandes, intolerancia de ambigüedad, hostilidad y contracontrol). Los resultados factoriales arrojan 4 factores significativos estadistica y psicológicamente. Se presentan las diferencias intergénero en total, por estructura de miedos y a lo largo de la edad (acercamiento transversal). El volumen de miedos por factor se mantiene constante en ambos géneros a lo largo del grupo de edad, las comparacionés intergénero sugieren que o bien no hay diferencias o aparecen algunas diferencias a favor de los hombres. Se discuten los compromisos con personalidad y las relaciones con neuroticismo no parecen ser las esperables, aunque existen razones para eilo. Los resultados se discuten en el contexto de normalidadpsicopatologla, asi como del valor wadaptativo» de los miedos y su estabilidad a lo largo del ciclo vital.

Palabras clave: MIEDOS, EVALUACIÓN DE MIEDOS, ESCALAS DE CALIFICACIÓN, MIEDOS Y PERSONALIDAD.

Correspondencia: vpelecha@ull.es.

El presente trabajo forma parte del proyecto SEJ2006-14301/PSIC Consolider, Bien-S-Star, aunque las opiniones vertidas en este trabajo son de exclusiva responsabilidad del autor y no compromete a ninguno de los otros miembros. 


\section{SUMMARY}

Structural, convergent and differential validity data are shown on a fear inventory for adults ( $N=576$ edults, $17-65$ years), both genders. Participants fulfilled also the big five personality factors (NEO-PI-R) and other three questionnaires (intolerance of ambiguity, hostility and counter-control). Factor analysis (varimax rotation from principal components) gave a four-factors solution; also the factor structure for each gender have shown that the structure is different for men and women. In the range of age of this study, the volume of fears is the same for all ages (transversal data), the differences between genders is not very great and in all comparisons, men score higher than women. Correlation results with personality are not totally coherent with the theoretical definition of the big-five. The results are discussed in relation with the literature published and the connections with personality and psychopathology.

Key words: FEAR, ASSESSWENT OF FEARS, RATNG SCALES OF FEARS, FEARSAND PERSONALTTY.

\section{UNAS ANOTACIONES ACERCA DE UNAASIGNATURA PENDIENTE: MIEDO, FOBIA Y ANSIEDAD}

Tradicionalmente y desde una perspectiva teórica se acepta que cuando se habla de miedo, se está tratando de una emoción básica y que posee componentes biológicos, motores y cognitivos (si un organismo no capta el objeto o entidad que genera miedo, el miedo no aparece), aunque no necesariamente deben ser contenidos conscientes'. Además, al miedo se le atribuye un valor adaptativo de conservación ante los peligros del entorno (al menos, por lo que se refiere a mamiferos, existen unos detectores con la función de detectar

\footnotetext{
' Se trata de componentes muy primarios y que no van en contra de la teoria motora de las emociones (estamos tristes porque lioramos) en la medida en que se tratarla de la detección de peligro; otra cosa sucede con el complejo de vivencias posteriores, después de haber csalidon de la situación de miedo.
} 
los peligros del entomo, unos patrones de respuesta de ataque o huida y unas expresiones faciales de miedo que pareen comunes a todas las culturas conocidas si se trata de seres humanos). $Y$, finalmente, aunque el miedo es un fenómeno general, se reconoce la existencia de diferencias individuales al menos en la expresión de esta emoción.

Tradicionalmente, en las cuestiones definitorlas (Pelechano, 1981, 1984; Sandin, 1997; Valiente, Sandín y Chorot, 2003) se hace mención de miedo, fobia y ansiedad. La apelación al Diccionario de la Real Academla Española (2001) no arroja mucha luz teórica u operativa².

Hace unos años, Barlow (1988) sugirio que mientras el miedo equivalía a una reacción de alarma primitiva ante la percepción de un peligro actual, unida a la conceptualización clásica de ataquehuida, la ansiedad es una combinación difusa cognitivo-afectiva, identificable como una aprensión ansiosa, que suele estar asociada a cualquler objeto, suceso o persona y que lleva consigo un enfoque de la atención hacia uno/a mismo/a. Esta propuesta harla depender el miedo de una respuesta «natural», mientras que la ansiedad seria el resultado de un aprendizaje (que bien puede ser de un solo intento), lo que oscurece, más que aclara el panorama al existir un gran volumen de miedos aprendidos que no serlan explicados de manera adecuada.

El meritorio trabajo de Gullone, King y Ollendick (2000) ha intentado encontrar y elaborar evidencia empírica a la propuesta de Barlow mediante el procedimiento de aislar patrones de covariación de respuesta a su cuestionario de miedos y aislaron tres factores: uno al que denominaron miedo, otro al que denominaron ansiedad y

\footnotetext{
2 El miedo es una «perturación angustiosa del ánimo, por un riesgo o daño real o imaginarios. Se enumera, además, arecelo o aprensión que alguien tiene de que le suceda algo contrario a lo que desean; la fobia es una waversión obseslve a algulen o atgos y tamblén, un ktemor irracional compulsivo*. Le ansiedad se identifica como un restedo de agitación, inquletud o zozobra del énimos y también, «angustia que suele acompanar a muchas enfermedades, en particular a clertas neurosis, y que no permite sosiego a los enfermosn. Estes definiclones, le verdad es que recogen poca o ninguna apeiación a procesos y entidades psicolígicas claras y además, expresan aiguna inexactitud (por ejemplo, Identificar la ansiedad con un cestado* en general, su asimilación a angustia y relacionarla con las cenfermedadess.La Identfficación de la fobla es mús pobre. Una vez más, seftelamos la necesidad de Incorporar al Diccionarto de le Academia las acepclones psicologicas de los términos utilizados y no apelar, exclusivamente (como en este caso) a significaciones emédicas" como se expresa en el Dlccionario menclonado.
} 
un tercero de experiencias fisiologicas. El hecho es, sin embargo, que la ansiedad no siempre llama a una activación indiferenciada sino incluso agresiva y con un patrón de actuación coherente y el miedo, a veces, lleva consigo, cuando es de gran intensidad, una inhibición comportamental rigida radical. En suma: las diferenciaciones propuestas son de alcance parcial y no han logrado una aceptación consensuada debido a sus insuficiencias teóricas y pragmáticas.

El Diccionario más reciente de psicologla ofrecido por la American Psychological Association (2007) es más explicito y recoge la mayor parte de lo que se encuentra en la bibliografla pertinente: El miedo es una kemoción intensa activada por la detecclón de una amenaza inminente, lleva consigo una reacción de alarma inmediata que dispara en el organismo un conjunto de cambios fisiologicos. Estos cambios incluyen aceleración del latido cardlaco, redirección del flujo sangulneo desde la penfferia hacia el interior, tensión muscular y una movilización general del organismo hacia la acción (...). De acuerdo con algunos teóricos, el miedo se diferencia de la ansiedad en que tiene un objeto (por ejemplo, un depredador, ruina económica) y es una respuesta proporcionada a la amenaza objetiva, mientras que la ansiedad, especificamente carece de un objeto o es una respuesta más intensa de la que es requerida por la amenaza percibidan.

La fobia es uun miedo persistente e irracional de una situación concreta, objeto $o$ actividad (por ejemplo, altura, perros, agua, sangre, conducir coches o volar en avión), que, consecuentemente, es o evitada con intensidad, o mantenida con intenso sufrimiento».

Finalmente, la ansiedad es uun estado de ánimo caracterizada por aprensión y síntomas somáticos de tensión en el que un individuo anticipa un peligro inminente, catástrofe o Infortunio. La amenaza futura puede ser real o imaginada, interna o externa. Puede ser una situación identificable o un miedo más vago de lo desconocido (por ejemplo, un sentido general de condena inminente). El cuerpo, a menudo se moviliza para hacer frente a la amenaza. Los músculos se tensan, la respiración se hace más rápida, y la tasa cardiaca se acelera. La ansiedad se puede diferenciar del miedo, tanto fisiológica como conceptualmente, aunque los dos términos son usados, erróneamente, de forma intercambiable». 
La diferencia básica, entre miedo-fobia y ansiedad es la indeterminación o inespecificación del ente que provoca las respuestas por un lado y, por otro, unas vías fisiológicas de activación que son, en parte, distintas. La «anticipación» de la respuesta de ansiedad puede encontrarse asimismo presente en el miedo y en la fobia (caso de conocer, más o menos, el nivel de peligro y «esperarlo»). La existencia de vias fisiológicas muy distintas en los tres fenómenos se anotan a veces, pero no siempre, debido a una cierta indiferenciación en el proceso de activación (distinto al comienzo pero que van convergiendo a medida que avanza).

Dos puntos de considerable interés en este punto se refieren a la "diferencia» entre miedo y fobia por un lado y a los procesos de transformación de un elemento en otro. La diferencia externa parece clara: hay fobia y ansiedad cuando existe psicopatología. El caso es que los criterios aducidos son la falta de relación entre estimulo y respuesta (cuando la respuesta sobrepasa, en mucho, a la que cabrla esperar en una situación «normal») y la persistencia de una reacción inadecuada ante un animal, objeto o situación que pervive más allá de un perlodo de tiempo que se estima entre 3 y 6 meses. El caso es que no resulta muy preciso apelar a esobrepasars a la reacción normalmente esperable y que en gran medida, la reacción se encuentra mediada por las tradiciones culturales (que no se encuentran presentes en la definición); por otro lado, la ufalta de relación» entre estímulo y respuesta tampoco es un criterio muy cientifico y cuantitativo: Ya en otra ocasión (Pelechano, 1984) haciamos referencia a la importancia que posee el contexto (que incluirla referentes al medio y a la persona, su nivel evolutivo, etc.) en el que vive el ser humano para poder enjuiciar el grado de adecuación o no de la respuesta en márgenes muy amplios: ante un mismo objeto o situación, estos contextos pueden permitir un rango considerable de respuestas dentro de lo que se considera «normal». Sucede, además, que puede haber emoción fuerte y junto a ello, una respuesta motora muy débil (o ninguna) como cuando nos encontramos ante un objeto fóbico y, sin embarga, podemos llevar a cabo conductas «normales», que entren en el rango de la normalidad o incluso que sean anormalmente esperables en condiciones normales (un elemento que se identifica con «valentla»). 
Siendo las tres expresiones parte de un mismo territorio semántico y siendo el criterio de diferenciación la adecuación de respuesta (en sus vertientes de duración, generalización y de intensidad) lo que en buena cuenta diferencia la normalidad de la anormalidad, deberían ser conocidos los procesos que permiten la aconversion» de uno en otro. Esta kconversión» no se presenta con el mismo valor y posibilidad para todos los casos (existen unos objetos y/o animales y/o situaciones para los cuales los humanos desarrollamos más respuestas de miedo, que en otros, por lo que se ha propuesto una epreparación» filogenética ante ellos) y, realmente, aparte alguna demostración experimental en estudio de caso, hoy en dla todavia nos encontramos lejos de conocer las variables y dimensiones o procesos psicolbgicos que definen estas transformaciones. Conocemos la eficacia de algunos procedimientos que han funcionado relativamente bien para la eliminación de fobias e incluso de ansiedades (acercamlentos de terapia cognitivocomportamental), pero que no necesariamente nos dan información acerca de la formación y transformación a la que se ha hecho mención más arriba. Y sucede que unos humanos presentan miedo ante unos animales, objetos o situaciones mientras que otros no parecen tenerto (incluso de animales para los que existirla esa «preparación» en el caso de mamiferos superiores, como sucede con las serpientes, a las que algunos en nuestros dlas las mantlenen como mascotas o animales de compañla): la sangre, heridas abiertas, cadáveres, muerte y simbolos de la muerte... son elementos comunes en unas profesiones y se encuentran ausentes en otras (al ser comunes, no deben ser objeto de miedo para los que tienen que ver con esas profesiones, con lo que la influencia soctocultural en miedos, fobias y ansiedad debe ser rotable). De ahí que la knaturalidad» de respuesta y la cadecuación» que aparentemente es de gran sentido común, deja de ser un criterio claro de identificación de unos fenómenos u otros a menos que se tomen en cuenta las variables que identifican los contextos de vida y que se incluyan en las definiciones de miedos, fobias y ansiedad. Es por este tipo de ausencias por to que, posiblemente, la psicologla tenga sin aprobar una pslcología aparentemente tan simple como la definición de estos conceptos y su interrelaclón dinámica. Y ello nos lleva al punto siguiente sobre estructura y evolución de los miedos. 


\section{LA ESTRUCTURA Y LA EVOLUCIÓN DE LOS MIEDOS}

\section{Acerca de los acercamlentos para delimitar la estructura de los mledos}

Si hubiese un modelo teórico omnicomprensivo y coherente, la estructura podria derivarse, en gran medida, de este modelo. El caso es que tal modelo no existe y en su ausencia, los problemas de estructura y, los derivados de aquí (la evolución) pueden dar lugar, en ocasiones distintas, a resultados que no son totalmente solapables entre si. Algunas consideraciones parecen tener interés en este punto.

En primer lugar, si se trata de elaborar inventarios de miedos, éstos deberian incorporar prácticamente todos lo objetos, situaciones y seres que pueblan el planeta y con los que tiene experiencia el ser humano. Cuando se llevan a cabo análisis encaminados a descubrir los "patrones» de covariación que existen, la metodologla utilizada al respecto (análisis de escalas o factorial como ejemplos paradigmáticos) posee limitaciones claras: alsia aquellos patrones que ofrecen, en la muestra de participantes que han cumplimentado la prueba, patrones que cumplen con los criterios estadisticos de representatividad (no quiere decir que sean los más relevantes, o clinicamente significativos), distribución y correlación. Con lo que si el abanico de elementos es amplio, deben perderse siempre aquellos que aun siendo relevantes, no cumplen estos criterios.

Por otro lado, los patrones que se aíslen (los factores, en su caso) estarán compuestos por los patrones de covariación encontrados, no necesariamente por la coherencia teórica intema de los items. O dicho con otras palabras: es posible que aparezcan factores en los que saturen elementos tales como serplentes, tigres, oscuridad, ir en avión, personas desconocidas y ataúdes. Este tipo de resultados sugiere que estos elementos tienen algo en común (obviamente la covariación entre ellos) y, desde ahi, cabe pensar que debe haber aalgo más» en común en un nivel de análisis conceptual y operativo, que le adé sentido», lo que no siempre resulta fácil.

Una opción alternativa consiste en «depurar» al máximo los elementos que forman los instrumentos de evaluación. Así, por ejemplo, muestrear algunos dominios (por ejemplo, animales domésticos, 
situaciones sociales nuevas y estresantes, peleas y discusiones) con el mismo o muy similar número de elementos y aplicar un análisis factorial confirmatorio acerca de la existencia de tres factores y elaborar un instrumento a partir de estos resultados ya depurados (obvlamente, con este procedimiento, asimismo se pierden aquellos elementos que no cumplen ciertos requisitos estadisticos, aunque la "claridad" es mayor, como mayor es la pérdida de información y la imposición de una estructura «racional» (o aplicación de un filtro que permita que aparezcan solamente estos elementos «claros»). La idea de estructura que se logra así es más clara, menos rica y más alejada de la realidad emplrica observable y se pierde más información aunque se gana mayor coherencia teónica en los resultados.

En nuestro caso, nos hemos decantado pro ampliar el horizonte definidor de los miedos, utilizando análisis factorial exploratorio sobre una muestra de 100 elementos.

Acerca de los Items que forman los Instrumentos de evaluación

Debido a los problemas definicionales a los que nos hemos referido más arriba, lo que se encuentra en los Instrumentos de evaluación al uso es una mezcla de eiementos muy diversos y el hecho de responder de manera afirmativa a la cuestlón (se tiene mledo o mucho miedo) puede querer decir que se trata de un miedo o de una fobia (incluso de ansiedad, en la medida en que ésta tiende a ofrecer una puntuación altas de miedos en muchos de los ítems). La «variedad» de elementos resulta una buena recomendación para el estudio de la amplitud de los miedos, pero, con ello, pueden encontrarse, asimismo presentes, procesos y elementos psicológlcos muy dispares entre sl. La falta de información acerca de la persistencia y gravedad de las respuestas, asl como de los contextos de vida en los que viven los participantes hace muy dificil cuando no imposible, afinar en la evaluación diagnóstica diferencial precisa acerca de si se trata de miedos, fobia o ansiedad . Y ello, por otra parte, ayuda a la heterogeneidad y complejidad de los factores que se aísten en cada caso.

En este trabajo se ha maritenido la riqueza y heterogeneidad de los elementos a sabiendas de la dificultad interpretativa que ello puede traer consigo. 


\section{La evoluclón de los mledos}

Se trata de un tema no exento de polémica. Por lo que se refiere a niños pequeños (hasta 9 años), Pelechano $(1981,1984$ ) encontró que el volumen total de miedos parecia mantenerse en todas las edades, aunque se obtenlan diferencias claras en lo que se mostraba miedo. Una tendencia clara era el paso de miedos fisicos (cada vez menos) a miedos sociales (cada vez más). Una posible base empirica de resultados disonantes posiblemente se encuentre en que se hayan estudiado uno u otro tipo de miedos.

En el caso de los adultos el tema adquiere una importancia especial en la medida en que se han terminado los principales aprendizajes relacionados con la vida emocional, los afectos tienden a estabilizarse en la medida en que los contextos de vida en los que desarrollan su actividad los humanos tienden a ser similares y a cambiar poco durante un perlodo largo de tiempo (que llegaria hasta las puertas de la jubllación y desde la segunda parte de la década de los velnte). La hipótesis general (Pelechano, 1981) es que, a menos que sean tratados especificamente, los miedos se mantendrian a lo largo de la mayor parte del ciclo vital adulto, en condiciones normales. $Y$ ello frente a otras opciones en las que se defenderla que el miedo tendria que ser menor a medida que se van fortaleciendo y consolidando las estrategias de dominio de situaciones que se darla a lo largo de este ciclo vital.

\section{MIEDOS Y PERSONALIDAD}

La relación entre miedos y personalidad ofrece, desde una nueva perspectiva, una forma de acercarse a la delimitación operativa d diferencias entre miedo y ansiedad, si estudlamos la manera de relacionarse los factores de miedos con las dimensiones de personalidad. De entre las dimensiones de personalidad, la «ansiedad» ha sido evaluada medlante cuestionarios (lo que facilitarla la obtención de relaciones en la medida en que miedos y ansiedad se evaluarlan en este caso con un mismo tipo de instrumentos de medida: autoinformes) y serla la que, de entre todas las propuestas, ofrecerla mayor volumen de relaclón (y serla positiva). Sus relaciones con otras dimensiones serla más discutible, aunque por lo que se refiere a 
intolerancia de ambiguledad deberlan encontrarse relaciones positivas (más miedo, más intolerancia), las relaciones con contracontrol serlan inesperadas $y$, finalmente, por lo que se refiere a los factores de hostilidad y malevolencla, las relaclones, si se dieran, deberlan ser más bien tenues aunque positivas.

De rechazo, el patrón de correlaciones encontrado podrian contrastar e iluminar, desde una nueva perspectiva las similitudes y diferencias entre ansiedad y miedos, por sus "compromisos» diferenciales con la personalidad, si bien habria que afladir que "ansiedad» psicopatológica y ansiedad evaluada mediante cuestionarios de personalidad no son términos sinónimos.

\section{OBJETIVOS DE ESTE TRABAJO}

Con este panorama de ideas, sugerencias y resultados, pueden establecerse los principales objetivos que persigue este trabajo:

(1). Aislar la estructura factorial de los miedos en jóvenes y adultos (edad de 17 a 70 años).

(2). Estudiar la evolución de los miedos en este rango de edad.

(3). Ofrecer datos respecto a la diferencia en miedos entre géneros dentro de este rango de edad. Estas diferencias se ofrecen en estructuras y en evolución de los afactores comunes».

(4) .Presentar resultados acerca de los correlatos de personalidad de los factores aislados de mledo.

El primer objetivo sirve como contrastación de la estructura de miedos y fobias que aparecen en la bibliografla, tanto en el plano global (ambos géneros), como particular (para hombres y para mujeres, en parte del tercer objetivo). El segundo objetivo tiene que ver con la entidad epistemolbgica de lo que se evalúan en las escalas-inventarios de miedos, así como con el problema de la estabilidad del volumen e intensidad de los miedos en esta parte del ciclo vital. Complementariamente los resultados acerca de las diferencias intergénero en miedos ofrecerá cierta luz respecto a los cambios que se están dando en la sociedad contemporánea. Finalmente, el cuarto objetivo ofrece las relaciones entre miedos y ciertas dimensiones de personalidad que poseen interés por si mismas y para anclar los miedos dentro de un esquema más global de funcionamiento personal. 


\section{MÉTODO Y PROCEDIMIENTO}

\section{Participantes}

El total de participantes en este estudio han sido de $611,73,6 \%$ de ellos, mujeres. La edad media ha sido de 25,27 años (desviación típica de 7,49 ) y un rango que va desde los 17 a los 70 años. Por lo que se refiere al estado civil, el $79,2 \%$ han sido solteros (aunque una quinta parte de ellos viviendo en pareja), un $8,3 \%$ estaban casados y un10, $5 \%$ eran viudos(as); la tasa de divorciados fue de 0,8 .

La mayorla no tenía hijos $(84,1 \%)$, un $3,8 \%$ tenla un hijo y el $2,7 \%$ dos.

Los estudios de los que informan han sido: el $4,7 \%$ tenlan todos o parte de estudios primarios, el $44,5 \%$ secundarios y el $50,6 \%$ universitarios (completos o parte).

Por lo que se refiere a nivel ocupacional, el $4,2 \%$ informan de encontrarse en estado de parado o jubilado, el $6 \%$ con trabajos manuales no cualificados; el $6,4 \%$ con trabajos manuales cualificados, el $5,4 \%$ son autónomos con pequeña empresa o administrativos; un $3 \%$ son profesionales liberales y un $75 \%$ están cursando estudios. Poco más del $80 \%$ reside en el área metropolitana de Santa Cruz de Tenerife-La Laguna (Tenerife).

\section{Instrumentos}

El inventario de miedos.- Se trata de un inventario que consta de 100 elementos entresacados y adaptados de un instrumento similar para estudiar la evolución de miedos en niños generado por Pelechano (1981, 1984) a partir del Fear Survey de Wolpe. El instrumento pide que se exprese el miedo que se tiene a una serie de elementos que forman la prueba. Existen tres posibilidades de respuesta: nada (0), algo (1) y mucho (2). El análisis racional de la prueba indica que está formada por los siguientes núcleos racionales. (1) miedo a animales de distinto tamaño y peligrosidad como serpientes, perros, gatos, arañas y ratas; (2) miedo a fenómenos naturales, básicamente metereologicos como tormentas, truenos y relámpagos; (3) miedo a daño corporal y enfermedades como heridas, enfermedades propias 
o de familiares; (4) miedo a muerte y símbolos de la muerte, como la muerte, ataúdes y ertierros; (5) miedo a relaciones personales hostiles y/o agresivas como peleas, discusiones entre miembros de la familia, que le chillen y amenazas, miedo a ladrones; (6) miedo a lugares cerrados como habitaciones cerradas, estar solo/a en casa y situaciones disparadoras de agorafobia como cruzar las calles; (7) enfermedades y referentes médicos como sangre, intervenciones quirúrgicas, verse sangrar una herida, inyecclones y enfermedades; (8) miedo a situaciones sociales como ser observado/a, que lo consideren tonto/a, cometer errores y reuniones con gente desconocida o entrar solo en un lugar extraño; (9) Miedos a viajar en coche, barco o avión (también miedo al agua); (10) pérdidas como perder el cariño de personas que se quiere, a perder regalos que se aprecian; (11) miedo a seres sobrenaturales o fantasiosos como películas de miedo, extraterrestres, OVNIS, fantasmas y (12) miscelánea en donde se encuentran personas deformes, la policia, los cuchillos.

El abanico de categorias representadas es amplio con el fin de poder ofrecer una gran variabilidad y recoger las pautas que existen en esa variabilidad.

Instrumentos de personalidad.- Los participantes han cumplimentado el NEO-PI-R de Costa y McCrae (1992) en la versión española (Avia, Sanz y Sánchez-Bernardos, 1997) y con las modificaciones que fueron introducidas por de Miguel y Pelechano (2000) en algunas formulaciones de ítems con el fin de alcanzar una mayor adecuación al significado original. El cuestionarlo, compuesto por 240 elementos, debe cumplimentarse eligiendo para elemento, una de cinco altemativas de respuesta: A (totalmente en desacuerdo, 0 ), B (en desacuerdo, 1), C (neutro o ni uno ni otro polo, 2), D (de acuerdo con lo que dice el ítem, 3) y $E$ (totalmente de acuerdo, 4). Alsla cinco factores: neuroticismo, extraversión, apertura, cordialidad y escrupulosidad, cada uno de los cuales se encuentra formado por seis facetas.

Cuestionario de hostilidad y malevolencia HOSTYMAL de Pelechano (2000), formado por 27 elementos que deben ser cumplimentado con dos altemativas de respuesta (verdadero y falso) y formado por tres factores: (i) dureza de trato con logro de fines al 
margen de las normas sociales, con ítems del tipo «El mejor modo de defensa es el ataque», «En cualquier caso, lo que se debe hacer es ganar a toda costa, sin dar demasiada importancia a las reglas de juegon; (ii) malevolencia y rencor hacia los demás y el poder político, con items del tipo «La mayoria de las personas son inteligentes y bondadosas» (corregido al revés) o "Cuando alguien me ha hecho una jugada sucia, lo mejor es olvidarlo» (corregido asimismo al revés) y (iii) desconfianza en el ser humano con ítems del tipo «El ansia de poder es vital en mucha gente" o «Mucha gente predica una cosa...y hace otra).

Cuestionario de contracontrol (CC) de Pelechano (Pelechano, Peñate y González, 1997) formado por 105 elementos y cuatro alternativas de respuesta (nunca, alguna vez, frecuentemente y siempre). Aísla tres factores de segundo orden: (i) contracontrol beligerante ante influencia externa de tipo social e institucional (por ejemplo: Hay que oponerse a cualquier ley que consideremos injusta» o No consiento que nadie pretenda decidir por mi»; (ii) exculpación ante fracaso e indecisión con aceptación de control externo, como "Yo hubiese llegado mucho más alto si hubiese tenido kpadrinos», 0 «Me dejo llevar mucho por las costumbres y opiniones de los que me rodean» y (iii) rechazo de influencia familiar con imposición de puntos de vista propios como en «Cuanto más discutimos en casa sobre to que tengo que hacer, más ganas me vienen de hacer lo que me de la gana», o Cuando yo sé bien lo que mi grupo de amigos/as tiene que hacer, me impongo a todos/as y decido ser el único que dará órdenes».

Cuestionario de intolerancia de la ambigúedad de Pelechano (Pelechano y Aguilera, 2004), formado por 64 elementos de dos alternativas de respuesta (verdadero y falso) y un solo factor de segundo orden. El referente de los ítems cubre los dominios del mundo personal, de ideas y creencias y socio-laboral.

\section{Modo de cumplimentación}

La cumplimentación de todas las pruebas fue indlvidual. En primer lugar, se tomó contacto con los alumnos de psicología de la personalidad de la universidad y se les pidió colaboración para un estudio en el que se estudiaban elementos relacionados con sabiduria, 
vida emocional y personalidad. A aquellos alumnos que deseaban colaborar se les recompensaba con un suplemento de puntuación (medlo punto en la parte de prácticas de la materia). Se entregaba el cuademillo de pruebas para que lo cumplimentaran en su casa. Una vez devueltos los instrumentos, se les ofrecla la posibilidad de que famlliares y amigos pudiesen cumplimentar las pruebas y se les entregaba cada uno de los cuadernillos en un sobre con el fin de que se lo entregaran a las personas que iban a cumplimentar y éstas o bien se los devolvian en sobre cerrado, o blen se remitlan directamente al Departamento de Personalidad, Evaluación y Tratamientos Psicológicos de la Universidad de La Laguna (Tenenife, Islas Canarias). Esta estrategia se utilizó en tres cursos consecutivos. La mayorla de los participantes cumplimentaron todas las pruebas y éstos son los que forman la muestra que ha sido analizada en este trabajo.

\section{LA ESTRUCTURA «GENERAL»DE LOS MIEDOS}

El inventario de 100 elementos de miedos recoge una amplia vaniedad de disparadores y/o creadores de miedos, desde elementos de experiencia cotidiana (animales domésticos, circulación, tormentas con aparato eléctrico), hasta sucesos vitales estresantes de gran intensidad como muerte de familiares y amigos, enfermedad grave, elementos que suscitan imágenes de muerte (ataúdes, entierros) y fenómenos imaginados (Ovnis, extraterrestres), asl como miedos sociales (hacer el ridiculo, sentirse observado/a, tener que hablar en público o caerse en públlco). Se trata de una revisión del instrumento que fue generado y aplicado en dos ocasiones para niños (Pelechano, 1981, 1984).

Se ha realizado un análisis factorial, con extracción de componentes principales y rotación varimax para maximizar la Independencia de los factores alslados. Este objetivo ha llevado asimismo a utilizar un criterio de selección de items duro: peso factorial igual o superior a 0,40 en un factor; en el caso de que hubiera un item con pesos de esta cuantla en más de un factor, era eliminado (no se dio ningún caso en estas circunstancias). A la hora de pedir extracción y rotación de factores se exigla un porcentaje de varianza total explicada igual o superior a 3 por 300 . 
La matriz resultante ha sido significativa y ha dado lugar a cuatro factores que explican el $31,56 \%$ de la varlanza total. La matriz factorial rotada en la que se incluyen solamente los items, con peso igual o superior a 0,40, se encuentran en la tabla 1. En la tabla se han elirninado los ceros y la coma decimal tanto en las saturaciones factoriales como en la comunalidad $\left(h^{2}\right)$.

Tabla 1.- Matriz factorial roteda de inventario de miedos. Extracción de componentes principales, rotación varimax sobre 100 items y 575 participantes

\begin{tabular}{|l|l|l|l|l|l|}
\hline Item & I & II & III & IV & $\mathrm{h}^{2}$ \\
\hline 1. Hablar delante de mucha gente & & .50 & & & 26 \\
\hline 2. La guerra & & & 42 & & 20 \\
\hline 3. Que alguien le ataque y le haga dafio en la calle & & & 44 & & 27 \\
\hline 4.Ver ataudes y coches fúnebres & & & & & 31 \\
\hline 5. Separarse de los familiates & & & 40 & & 21 \\
\hline 6. Los extraterrestres & 48 & & & & 25 \\
\hline 7. Que me humillen o se burlen de mí & & 59 & & & 40 \\
\hline 8. Los OVNIS & 49 & & & & 26 \\
\hline 9. Lugares donde ha muerto alguien & 47 & & & & 37 \\
\hline 10. Que el profesor (el jefe) me pregunte en clase (lugar de trabajo) & & 54 & & & 32 \\
\hline 11. Los dentistas & & & & 49 & 27 \\
\hline 12. Los gatos & & & & & 17 \\
\hline 13. Las cuevas & 50 & & & & 28 \\
\hline 14. Las armas & & & & & 25 \\
\hline 15. Los borrachos & & & & & 22 \\
\hline 16. Fracasar & & 42 & & & 32 \\
\hline 17. Una habitación desconocida & 50 & & & & 29 \\
\hline 18. Presenciar peleas familiares & & & & & 30 \\
\hline 19. La sangre & & & & 63 & 46 \\
\hline 20. Personas que tienen mal genio & & & & & 22 \\
\hline 21. Las ratas & & & & & 23 \\
\hline 22. Los gusanos & 47 & & & & 27 \\
\hline 23. Las peliculas de terror & 44 & & & & 27 \\
\hline 24. Montar en bicicleta & & & & & 06 \\
\hline 25. Los relámpagos & 66 & & & & 47 \\
\hline 26. Que me ignoren & & 53 & & & 35 \\
\hline 27. Estar entre mucha gente & & 44 & & & 24 \\
\hline 28. La oscuridad & 53 & & & & 35 \\
\hline 29. Ver poner inyecciones & & & & 69 & 51 \\
\hline 30. Que le peguen & & & 41 & & 29 \\
\hline 31. Perderse entre la gente & & 42 & & & 36 \\
\hline 32. Ver heridas abiertas & & & & 57 & 49 \\
\hline 33. Los entierros & & & & & 37 \\
\hline 34. Los módicos & & & & 48 & 29 \\
\hline 35. El fuego & 51 & & & & 32 \\
\hline
\end{tabular}


Tabla 1.- Matriz factorial rotada de inventario de miedos. Extracción de componentes principales, rotación varimax sobre 100 ftems y 575 participantes (continuación)

\begin{tabular}{|c|c|c|c|c|c|}
\hline 36. Las personas deformes & 41 & & & & 23 \\
\hline 37.Ser castigaio por Dios & & & & & 20 \\
\hline 38. Amerazas de otras personas & & & & & 32 \\
\hline 39. Perder el cariffo de las personas que más quiere & & & 58 & & 39 \\
\hline 40. Los túneles y pasadizos & 55 & & & & 36 \\
\hline 41. Ser atacado por animales & 40 & & & & 32 \\
\hline 42.Conpeter enrores & & 55 & & & 37 \\
\hline 43.Presenciar peleas en ba calle & 42 & & & & 34 \\
\hline 44. Reuniones con personas desconocidas & & 60 & & & 40 \\
\hline 45. Entrar solohe en un hugar extrofto & & & & & $\overline{34}$ \\
\hline 46.Perder objetos queridos & & & 41 & & $\overline{35}$ \\
\hline 47. El mar & 42 & & & & 19 \\
\hline 48.Cacrme al suelo & & & & & $\overline{34}$ \\
\hline 49. Los truenos & 67 & & & & 50 \\
\hline 50. Viajar en avión & 45 & & & & 24 \\
\hline 51. Ve persoass muertas & & & 46 & & 41 \\
\hline 52. Ver hospitales y elinicas & & & & 43 & 29 \\
\hline 53.Entrar en uns reunión donde ya eat la gente sentada & & 61 & & & 41 \\
\hline 54. Ver animales muertos & & & & & 26 \\
\hline 55. Ver maltratar a ctras personas & & & 49 & & 37 \\
\hline 56. Las habibciones pequeflas y cerradas aunque sean conocidas & & & & & 15 \\
\hline 57.Crueer las calles & & & & & 22 \\
\hline 58.Los bdrones & & & & & 33 \\
\hline 59.Las ranas & & & & & 17 \\
\hline 60.Estar enferma/a & & & & & 17 \\
\hline 61.Las serpientes & 41 & & & & 28 \\
\hline 62.Las arafias & & & & & 23 \\
\hline 63.Los ruidos fuertes & 48 & & & & 37 \\
\hline 64.10a cementerios & 43 & & & & 38 \\
\hline 65. Que me priten & & 46 & & & 32 \\
\hline 66.Lo fantasmas & 43 & & & & 26 \\
\hline 67. La mucrte de mis pedres & & & 70 & & 50 \\
\hline 68E El acentor & & & & & 19 \\
\hline 69.Estar solo/a en casa & 46 & & & & 28 \\
\hline 70.Tomar modicinas & & & & & 15 \\
\hline 71.Verme sangrar una berids & & & & 63 & 47 \\
\hline 72.Los perros & & & & & 19 \\
\hline 73.Fstar en lugares altos & & & & & 13 \\
\hline 74.Que me pongan inyecciones & & & & 77 & 61 \\
\hline 75.Que me saquen sangre & & & & 78 & 61 \\
\hline 76.0ue me critiquen, que hablen mal de mi & & 66 & & & 50 \\
\hline 77 Las cucarachas & & & & & 19 \\
\hline 78.Los espiritus & 47 & & & & 36 \\
\hline 79.Ser sometido/a a iotervenciones quinúrgicas & & & & & 26 \\
\hline 80.La muerte & & & $\mathbf{5 2}$ & & 35 \\
\hline
\end{tabular}


Tabla 1.- Matriz factorial rotada de inventario de miedos. Extracción de componentes principales, rotación varimax sobre 100 items y 575 participantes (continuacion)

\begin{tabular}{|c|c|c|c|c|c|}
\hline 81.La universidad (el hugar de trabajo) & & 41 & & & 22 \\
\hline 82.La suciedad & & & & & 17 \\
\hline 83. Que me consideren tonto & & 65 & & & 47 \\
\hline 84.Ser observado/a & & 70 & & & 55 \\
\hline 85.Las personas desconocidas & & 52 & & & 40 \\
\hline 86.Sentirse rechazado/a & & 64 & & & 50 \\
\hline 87. La muerte de hermanos & & & 70 & & 52 \\
\hline 88. Mirar hacia abajo en los lugares altos & & & & & 15 \\
\hline 89. Viajar en coche & 40 & & & & 20 \\
\hline 90.La policía & & & & & 16 \\
\hline 91.Los cuchillos & & & & & 23 \\
\hline 92. Viajar en barco & 42 & & & & 22 \\
\hline 93.Los caballos & 40 & & & & 18 \\
\hline 94.Los dentistas & & & & 49 & 27 \\
\hline 95.Las tormentas & 69 & & & & 52 \\
\hline 96.Ser observado/a mientras trabajo & & 63 & & & 44 \\
\hline 97.Enfermedad de mis padres & & & 67 & & 49 \\
\hline 98. Muerte de amigos/as & & & 70 & & 52 \\
\hline 99. Historias de miedo & 50 & & & & 37 \\
\hline 100 . A agua & & & & & 14 \\
\hline
\end{tabular}

Nota.- Se han omitldo los ceros y la coma decimal. Se ha redondeado a dos decimales. El criterio de selección de items por saturación era una saturación en un factor tgual o superior a 0,40 y que no saturara en esa cuantía en otro factor. Caso de saturar en más de un factor con esa cuantía se eliminaba el item.

Casi una tercera parte de los items (31) no cumplen con los criterios. $Y$, posiblemente resulta de cierto interés señalar lo que no parece estar pautado de forma coherente para la muestra participante.

El primer grupo de elementos se refiere a animales, domésticos (gatos, perros) y otros que no lo son (ratas, ranas y arañas). En algunos casos posiblemente por alergias, en otros, por asco. El segundo grupo tiene que ver con la muerte: ataúdes y coches fúnebres, entierros y ver animales muertos. El tercer grupo recoge situaciones de salud/ enfermedad, como estar enfermo/a y las intervenciones quirúrgicas. El cuarto, se refiere a elementos que tiene que ver con agorafobia como ascensores, habitaciones pequefias y cerradas, aunque conocidas, estar solo/a en casa, estar en lugares altos, mirar hacia abajo desde lugares altos. Un quinto grupo incluye ciertas personas como borrachos, ladrones y policla. El sexto grupo con violencia y 
amenazas, como armas, cuchillos, ser castigado por Dios y contemplar peleas familiares. Un último grupo, miscelánea con miedo al agua y a la suciedad. Aunque no parecen existir razones de tales resultados, en muchos casos las medias son altas (por encima de 1,80 sobre una puntuación máxima de 2) como sucede con la policla, estar solo/a en casa, cruzar las calles, las ranas y el agua y en algún caso la media es inferior a 0,90 como en el caso de las intervenciones quirúrgicas. En unos casos se trata de animales y objetos comunes y en otros casos precisamente porque no lo son, no presentan un patrón de covariación común y lo suficientemente potente como para entrar entre los «miedos comunes». Se tratarla de elementos que pueden generar miedos concretos pero que no poseen el grado de comunidad con otros que permita aislarlos de manera significativa en uno o más factores potentes. Pueden tener, con todo, una importancia clínica considerable precisamente porque tienden a no ser frecuentes y formando pauta con otros.

Vayamos ya con los factores aislados con el procedimiento que hemos utilizado.

El primer factor rotado está representado por 28 elementos, con un valor propio (y porcentaje de varianza total explicada) de 19,73. Los elementos se refieren a fenómenos meteorológicos y naturales (como fuego, sonidos fuertes, truenos, relámpagos, tormentas, el mar), fenómenos extrańos (como ovnis, fantasmas, espínitus, historias de miedo y peliculas de terror) y clertos elementos que hacen pensar en elementos con valor psicopatologico de agorafobia (como cuevas, habitación desconocida, lugares donde ha muerto alguien) o fobias de distinto contenido (como viajar en avión, viajar en barco, viajar en coche, la oscuridad), lo que se acompańa con percepción de amenaza a la integridad flsica como ser atacado/a por animales, los caballos o los gusanos. Se trataría de un factor complejo de miedo a ataques $y /$ o lesiones fisicas, junto con componentes simbólicos promovidos por la sociedad contemporánea y/o los medios de comunicación. Posee una consistencia interna muy alta (alfa de 0,91).

El segundo factor está representado por 18 items, con un valor propio de 5,00 (el mismo valor en cuanto a varianza total explicada). Se trata de un factor que podría identificarse en el caso de patología, de fobia social. Se trata de miedo social claro, con items del tipo de 
tener miedo a ser observado/a, a personas desconocidas, que le griten, reuniones con personas desconocidas, que le humillen o se burlen, a fracasar, sentirse rechazado/a, etc. La consistencia interna del factor es de 0,89 .

El tercer factor se encuentra representado por 13 elementos, con un valor propio de 3,66. Los contenidos de los Items cubren el miedo a ataques, la guerra, ver maltratar a personas, la muerte, la muerte de familiares y la pérdida del cariño de seres queridos. Se trataría de un factor de miedo a la muerte, agresí́n y pérdida de seres queridos. La consistencia interna (alfa de Cronbach) es de 0,84.

El cuarto factor se encuentra muestreado en la solución factorial por 10 elementos que cumplen los requisitos. Con un valor propio ( $y$ porcentaje de varianza total explicada) de 3,14. La consistencia interna (alfa de Cronbach) ha sido de 0,83. El contenido de los elementos sugiere que se trata de un factor de miedo a enfermedades, heridasy sangre (elementos relacionados con prácticas sanitarias).

\section{LA «EVOLUCIÓNミ DE LOS MIEDOS}

En un primer acercamiento para poner a prueba las hipótesis altemativas (si el miedo se mantiene constante a lo largo de las edades o dlsminuye con el incremento de las habilidades de dominio y el control personal que suele ir aparejado con la edad a menos que aparezcan procesos de deterioro). Hemos llevado a cabo un estudio transversal agrupando a los particlpantes en tres grupos de edad': de menos de 25 años (época en la que se tiende a estar «en formación»o los primeros intentos de búsqueda de independencia económica; un segundo grupo estará constituido por la fase de consolidaclón y logro de la independencia, junto con la estabilización de relaciones afectivas y personales y un tercer grupo de 40 a 60 años (existen algunos casos, 4, mayores a 60 años, y se han agrupados aqul) en donde se

\footnotetext{
${ }^{3}$ En buene lid, se deberla utllizar la edad afuncionaly mas que la cronológica en la medida en que lo que Importe en psicologla es lo que se hace o no en determinado perlodo de tiempo más que el mero paso del tempo (el paso del tempo serla indicador de eded cronológica). En eusencla de tales sededes funclonaless, se uthliza la eded cronológica suponiendo que ef cumplimlento de afios va aparejado con experienclas y depuración de modos de ver la realidad (no siempre, obvlamente, a mejor).
} 
encuentran ya los principales logros consolidados y se comienza el proceso de una cierta desaceleración laboral y familiar.

En la tabla número 2 se encuentran recogidas las medias obtenidas por cada grupo en cada uno de los factores, con expresión del número de participantes en cada grupo de edad.

Tabla 2.- Evolución de los miedos en la fase adulta para hombres y mujeres agrupados. Diferenclas por edad. La explicación en el texto

\begin{tabular}{|l|l|l|l|}
\hline FACTORES & $<25$ ANOS & 26-39 ANOS & $40-60$ AÑOS \\
\hline $\begin{array}{l}\text { M1. Ataques y lesiones, con componentes } \\
\text { simbólicos (seres metaforicos) }\end{array}$ & $41,50(433)$ & $41,99(145)$ & $41,45(33)$ \\
\hline $\begin{array}{l}\text { M2.Miedo social, ridiculo y hablar en } \\
\text { público }\end{array}$ & 23,56 & 24,01 & 23,06 \\
\hline M3. Muerte, agresión, perder carifio & 7,40 & 7,94 & 8,45 \\
\hline $\begin{array}{l}\text { M4.Hospitales y prácticas médicas } \\
\text { agresivas }\end{array}$ & 15,77 & 15,55 & 14,69 \\
\hline
\end{tabular}

Se han llevado a cabo pruebas de contraste de diferencias de medias (pruebas $t$ ) y no se han encontrado diferencias estadisticamente significativas para cada uno de los factores. Este resultado serla una prueba de la uconstancia» de los miedos a lo largo de periodos temporales largos y que cubnirla la mayor parte de la vida de los adultos.

Caso de apuntar algún cambio (si bien muy tenue y nada significativo) habrla que señalar que el miedo a la muerte, agresión y pérdida del cariño de los seres queridos tiende a incrementarse con el aso del tiempo y precisamente la tendencia contraria parece detectarse en el caso del miedo a enfermedades, heridas y sangre.

\section{LAS DIFERENCIAS INTERGÉNERO Y SU INTERACCIÓN CON LA EDAD CRONOLÓGICA}

Dos puntos, al menos, representan focos de interés. El primero se refiere a los problemas de estructura, el segundo, a la interacción y diferencias, en su caso, entre hombres y mujeres en miedo a lo largo del ciclo vital de la fase adulta.

Sobre la diferencia en estructura de los miedos en hombres y mujeres.- A pesar de un fondo común respecto al valor supervivencial y adaptativo que poseen los miedos en los dos géneros, es posible 
pensar que la manera de «ordenar» la realidad de los miedos pueda ser distinta en ambos géneros. Las razones que pueden aducirse al respecto van desde predominio hormonal, diferente en cada caso, hasta el peso diferencial que, todavia en nuestros dias, actúa en cada género.

En principio, dado el trato similar respecto a los «miedos» que se dan en nuestra cultura para ambos generos, cabrla esperar una estructura idéntica en hombres y en mujeres respecto a la manera de ragrupar" los miedos. Caso de que no se alcance esa identidad, el resultado abrirla una linea de trabajo que hasta ahora no se ha sustanciado empliclcamente y las explicaciones ofrecidas son postdictivas del fenómeno.

La aparición de diferencias, por otro lado, es posible que sean debidas a diferencias sustantivas (esto es, factores claramente distintos en cada caso, sobre el mismo material de prueba), o bien diferencias no sustantivas (el «salto», por ejemplo, de algún Item o su sustitución de un factor por otro Item que recogiera, la misma categoria, como por ejemplo «miedo a los gatos» frente a «miedo a las ratasn). En el caso de encontrar esas diferencias no sustantivas, podrían ser achacadas incluso a un sesgo en la elección de los items de cada instrumento pero que se mantendría sustantivamente, la misma estructura.

Por otra parte, la manera de poner a prueba si se trata de una estructura similar o no, puede hacerse de dos formas distintas, al menos. La primera, a partir de un análisis confirmatorio eligiendo como patrón de comparación la estructura encontrada en uno de los dos géneros (el de las mujeres por ejemplo). Con este procedimiento pueden compararse las estructuras si bien cuando el número de factores el superior a dos y la uentidad" y determinación de estos factores no está muy claramente definida, los resultados no siempre son aceptados por todos lo investigadores.

La segunda forma es más xprimitiva» aunque más cercana a los «datos reales" y nos permite acercarnos a los resultados factoriales obtenidos en cada caso: llevar a cabo análisis factoriales exploratorios independientemente para hombres y para mujeres y además, en la exposición, apuntar a las diferencias si las hubiere. Repárese que con ello no se pone en duda la estructura «común» (que sería 
necesaria para llevar a cabo los anállsis diferenciales por género y edad a lo largo del ciclo vital estudiado. Para ello, además, el tipo de extracción y rotaclón pedidas en cada caso deberla ser el mismo, como deberia ser utilizado el mismo material y forma de cumplimentación y, finalmente, deberian ser idénticos los criterios asumidos para la selección de los ítems en cada solución factorial. $Y$ esto es lo que hemos llevado a cabo como un primer acercamiento a la delimitación del problema. Se han llevado a cabo sendos anáiisis factoriales exploratorios, con petición de extracción de 4 factores (este ha sido el número de factores elegido para la estructura factorial conjunta), con extracción de componentes principales y posterior rotación varimax. Se han conservado solamente aquellos elementos con un peso factorial igual o superior a 0,40 en uno solo de los factores aislados.

(A). La estructura factorial de las mujeres ${ }^{4}$. Han participado un total de 425 y se ha factorizado la prueba entera (100 elementos) con el fin de poder apresar, si se daba, el fenómeno de «red undancia funcional» al que nos hemos referido más arriba. Los resultados obtenidos han sido los siguientes:

- A.1 Primer factor rotado: formado por 16 elementos, con un valor proplo de 18,61 y una varianza total explicada del $17,68 \%$. Se ha denominado, por el contenido de los ltems que lo definen como de miedo a animales, fenómenos meteorológicos, componentes de agorafobia (cuevas, túneles y pasadizos) y viajes en barco y avión.

-A.2. Segundo factor. definido por 18 elementos, con un valor propio de 5,38 y una varianza total explicada del $5,67 \%$. Claramente se trata de un miedo social (fracasar, hablar en público, cometer errores, desconocidos, etc.).

- A.3. Tercer factor: definido por 11 elementos, con un valor propio de 3,6 y una varianza total explicada del $3,68 \%$. Claramente se trata de un factor de miedo al daño fisico (amenazas incluidas) junto a

\footnotetext{
- Las correspondientes estructuras factorlales constan en poder del autor, y se puede tener acceso a ellas mediante petición. No se Incluyen en le trabajo para evtter el incremento de tablas numericas. Es verded que la diferencla en número de participantes es Importante y que la rolación entre número de variables y de participantes en el caso de los hombres podia apuntar a una lnestabilidad en la estructura de estos. Los criterios de selección de ltems, por otro lado, son bastante exigentes como pera entrar en la exposictón de resultados.
} 
enfermedad y muerte de familiares. Se trataría de un factor de miedo a la pérdida (a la integridad fisica, a la muerte de seres queridos $y$, antes, a enfermedad).

-A.4. Cuarto factor. Cubierto por 8 elementos, con un valor propio de 3,22 y un $3,39 \%$ de varianza explicada. Cubre con claridad el miedo a médicos, sangre y heridas, asi como Inyecciones.

(B). La estructura factorial de los hombres. Han participado un total de 151 hombres y los resultados se han sometido asimismo a análisis factorial con extraccion de componentes principales, 4 factores y rotación varimax, con un limite inferior en saturación de los ftems en los factores extraidos de 0,40 . Estos son los resultados:

- B.1. Primer factor. Con un valor propio de 21,26 y un $22,49 \%$ de varianza total expllcada. Se encuentra representado por 25 elementos. Se trata de un factor de miedo a fenómenos meteorológicos, a la oscuridad (y no cuevas ni pasadizos) y ataque de animales Junto a miedo social (desconocidos, que le consideren tonto, ser observado) y a actos médicos (incluido en su caso, la extracción de sangre).

- B.2. Segundo factor. Con un valor propio de 5,67 y un $5,32 \%$ de varianza total explicada. Está representado por 14 elementos. Se trata de un factor de miedo a la muerte y sus símbolos (ataúdes, coches fúnebres, entierros, cementerios) unido a actos médicos y miedo a integridad fisica (sangre, ver sangrar, sacarle sangre, ponerle inyecciones), a lo que se une viajes en avión y en coche.

- B.3. Tercer factor. Se encuentra representado por 16 elementos, con un valor propio de 3,79 y un $3,87 \%$ de varianza total explicada. Se unen en este factor componentes de miedo social (rechazo, fracaso, que le ignoren) junto a amenaza de perdida de seres queridos (muerte de padres y hermanos, enfermedad de famillares, e incluso estar enfermo).

- B.4. Representado por 10 elementos, tiene un valor propio de 3,11 y un $3,27 \%$ de varianza total explicada. Se trata de miedo a seres extranios (extraterrestres, OVNIS, gente deforme) aunque también ver hospitales y al agua.

El análisis de los elementos comunes suglere con claridad que la "redundancia funcional" no tiene una base empirica sólida en la delimitación de la estructura factorial (aparece en menos del $5 \%$ de elementos). En dos de los cuatro factores (el primero y el tercero), 
aunque no Idénticos, pueden rastrearse elementos comunes (en el primero, el $52,95 \%$ de elementos y en el tercero, el $43,7 \%$ de elementos). En estos dos factores, las diferencias más importantes entre ambos géneros se debe al papel que desempeña el miedo social; en el primero porque no se encuentran elementos de miedo social en el caso de las mujeres y sí en el de los hombres; en el tercero, porque de nuevo, elementos de miedo social se encuentran presentes en los hombres (que lo ignoren, sentirse rechazados, perder el cariño de seres queridos) y no se encuentra presente en las mujeres (isic). Da la impresión de que las cuestiones de relaciones personales se encuentran más «diversificadas" en los hombres (dos factores) que en las mujeres (un solo factor que las agrupa todas). Y este resultado apuntaria a una estructura más «claran y firme por parte de las mujeres que de los hombres en los miedos ( $y$, de rechazo, en la estructuración de la vida emocional). Si esto es específico de la muestra estudiada (toda ella habitantes de Canarias) o es un fenomeno de alcance más general, es algo que no puede decidirse con los resultados que hemos obtenido nosotros, aunque merecería un estudio de mayor alcance. Si parece que la edad no desempeña papel relevante en la medida en que el rango de edad en ambas muestras (hombres y mujeres) ha sido el mismo.

\section{La interacción género-edad en la evolución de los miedos}

Dada la diferente estructura encontrada en hombres y en mujeres, se ha optado por elegir la estructura general (en donde existe un predominio de mujeres, como se ha visto en la descripción de la muestra) y llevar a cabo los contrastes en su consideración evolutiva (en los tres grupos de edad). Los resultados se encuentran recogidos en la tabla número 3.

En la tabla el valor de las medias, el número de participantes en cada grupo (entre paréntesis). Los asteriscos indican que la diferencia correspondiente entre hombres y mujeres para el mismo grupo de edad es estadisticamente significativa (con el correspondiente nivel de significación indicado por asteriscos). Los principales resultados obtenidos son los siguientes:

(a). Aunque no siempre de forma estadisticamente significativa, en todos menos en un caso (factor cuarto de miedo a enfermedades, 

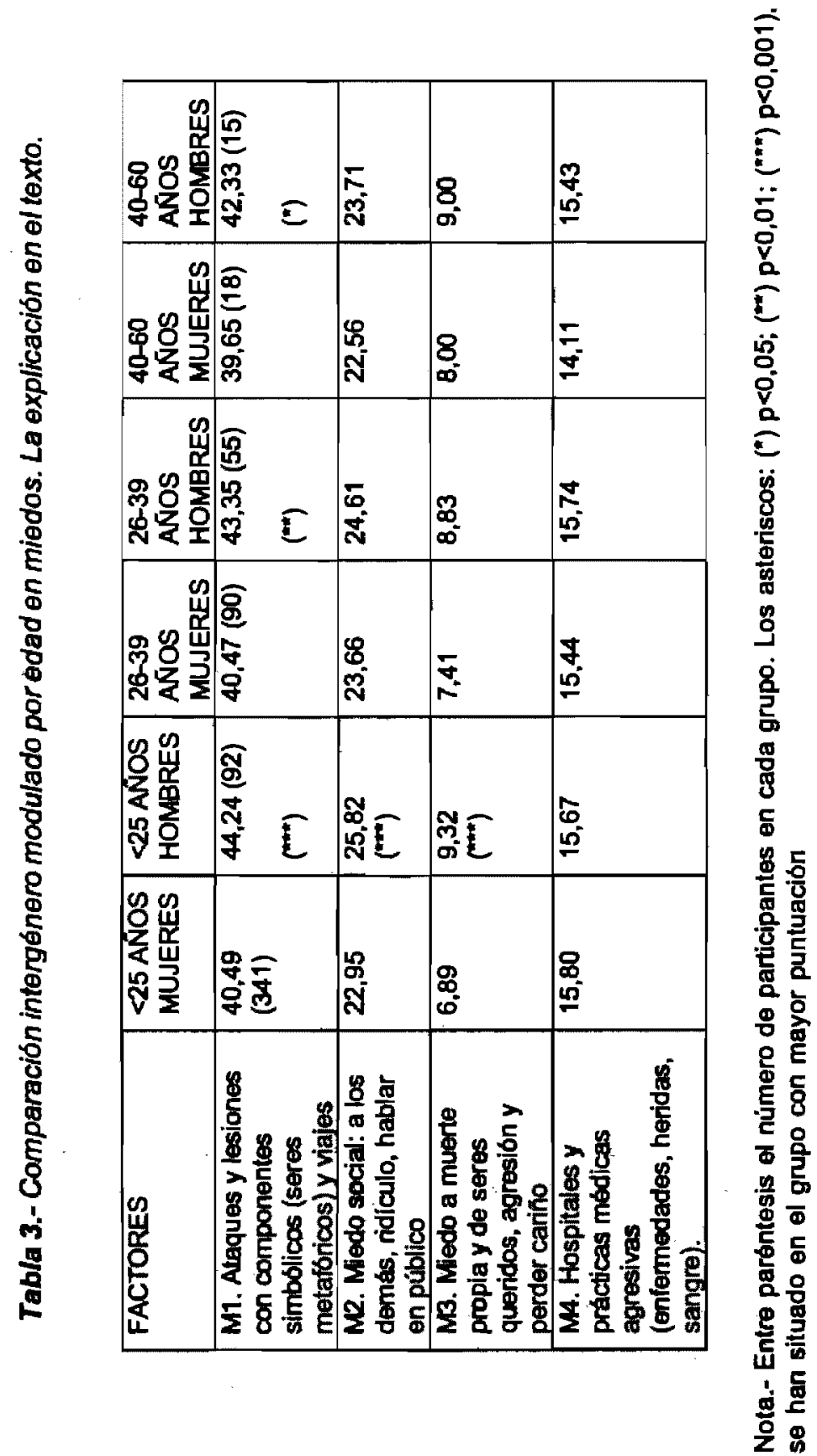
heridas y sangre y comparación en grupo de personas más jóvenes) los hombres puntúan más alto que las mujeres.

(b). El factor en el que todas las diferencias son estadisticamente significativas (más puntuación en los hombres) es el primer factor, recordamos que se trata de un factor complejo en el que se temen ataque físicos y lesiones, junto a seres fantásticos (fantasmas, extraterrestres, OVNIS) y fenómenos naturales (tormentas). Bien es verdad que firmeza de estas diferencias tienden a ir disminuyendo a medida que se avanza en edad.

(c). En el segundo factor (miedo social) y en el tercero (muerte, agresión y pérdida de seres queridos) se detecta una diferencia significativa a favor de los hombres solamente en el primer grupo de edad (menos de 25 años), después de lo cual, las diferencias desaparecen.

\section{LOS CORRELATOS DEL MIEDO: MIEDO Y PERSONALIDAD}

172 participantes de toda la muestra cumplimentaron todos los instrumentos y con toda la información de identificación. Esta submuestra estaba compuesta por un $27 \%$ de hombres, con una edad media de 26,61 años (desviación típica de 9,0), lo que no da resultados significativamente distintos del grupo total (y lo mismo puede decirse del resto de variables de identificación. En la tabla número 4 se encuentran los coeficientes de correlación (Pearson) entre los cuatro factores de miedo y los factores de personalidad.

En general los resultados correlacionales encontrados son más bien pobres y no se encuentran distribuidos de forma aleatoria. De los cuatro factores de miedos, es el que aglutina el miedo social el que presenta coeficientes de correlación mayores, en segundo lugar el factor tercero de muerte y agresión (la mitad de coeficiente superiores a .20 que en el caso anterior) y luego el cuarto factor de miedo a enfermedades heridas y sangre $y$, finalmente, el primer factor de ataques, lesiones y tormentas. De todas las dimensiones de personalidad evaluadas, es el factor de neuroticismo el que ofrece mayores coeficientes de correlación con miedos, y en todos los casos, los coeficientes son negatlvos ( $j$ sicl). El mayor corresponde a neuroticismo y miedo social. 


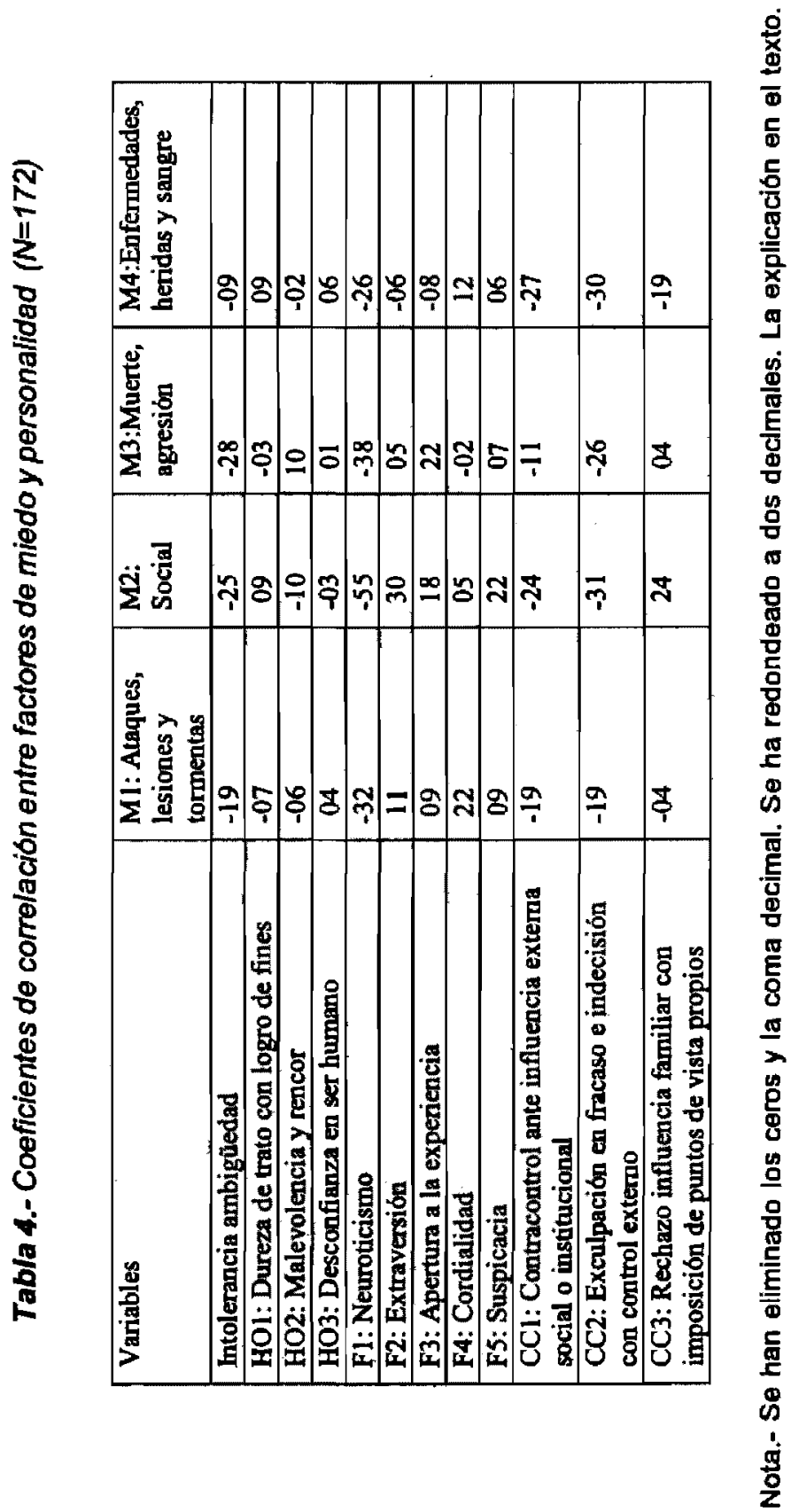


Asimismo, los cuatro coeficientes de correlación entre el segundo factor de contracontrol (exculpación en fracaso e indecisión) son negativos y tres de ellos superiores a 0,20 . Parece que a mayor exculpación e indecisión, menor volumen de miedos en todos los factores y más en miedos sociales por un lado y en miedo a enfermedades, heridas y sangre.

Otra variable presenta resultados que siguen esta línea: la intolerancia a la ambigüedad. A mayor volumen de miedos, menor intolerancia a la ambigüedad y esta tendencia es más intensa en miedo social y en miedo a muerte y agresión.

Un resultado que parece sorprendente es el que corresponde a miedo social y extraversión $(0,30)$. Este coeficiente indicarla que a mayor extraversión, mayor miedo social expresado, aunque la cuantía del coeficiente sugiere menos de un $10 \%$ de varianza común.

El factor de cordialidad de los cinco grandes presenta una relación significativa aunque tenue con el primer factor de miedos (ataques, lesiones $\mathrm{y}$ tormentas) y el factor de suspicacia tiende a favorecer un mayor miedo social.

Finalmente, sobre estos datos se ha llevado a cabo un análisis factorial (rotación varimax sobre componentes principales) con el fin de poner de manifiesto los patrones de covariación entre las variables. La prueba de esfericidad de Bartlett ha sido significativa $(p<0,0009)$ y se han obtenido seis factores con un valor propio igual o mayor a 1,0000 , con un porcentaje total de varianza explicada del $69,67 \%$. La matriz factorial rotada (varimax) con inclusión de pesos factoriales $<0,30$ y la comunalidad se encuentra recogida en la tabla 5 .

El primer factor tiene un valor propio de 3,70 (corresponde a un $23,10 \%$ de varianza explicada) y es el más potente. La inspección de los pesos factoriales en la tabla sugiere que se trata de un factor de contracontrol (saturan alto los tres factores de contracontrol) y de intolerancia de la ambigüedad $(0,62)$ y esto se acompaña con un rechazo de dureza de trato y logro de metas al margen de las reglas $(-0,54)$. Parece que se trata de un factor de rechazo de ambigüedad y de imposición de reglas y modos de pensar por parte de la familia y otros agentes sociales.

El segundo factor tiene un valor propio de 2,05 (un porcentaje de varianza total explicada de 12,82). Se encuentra definido por los cuatro factores de miedo aislados por el instrumento que presentamos $y$, 


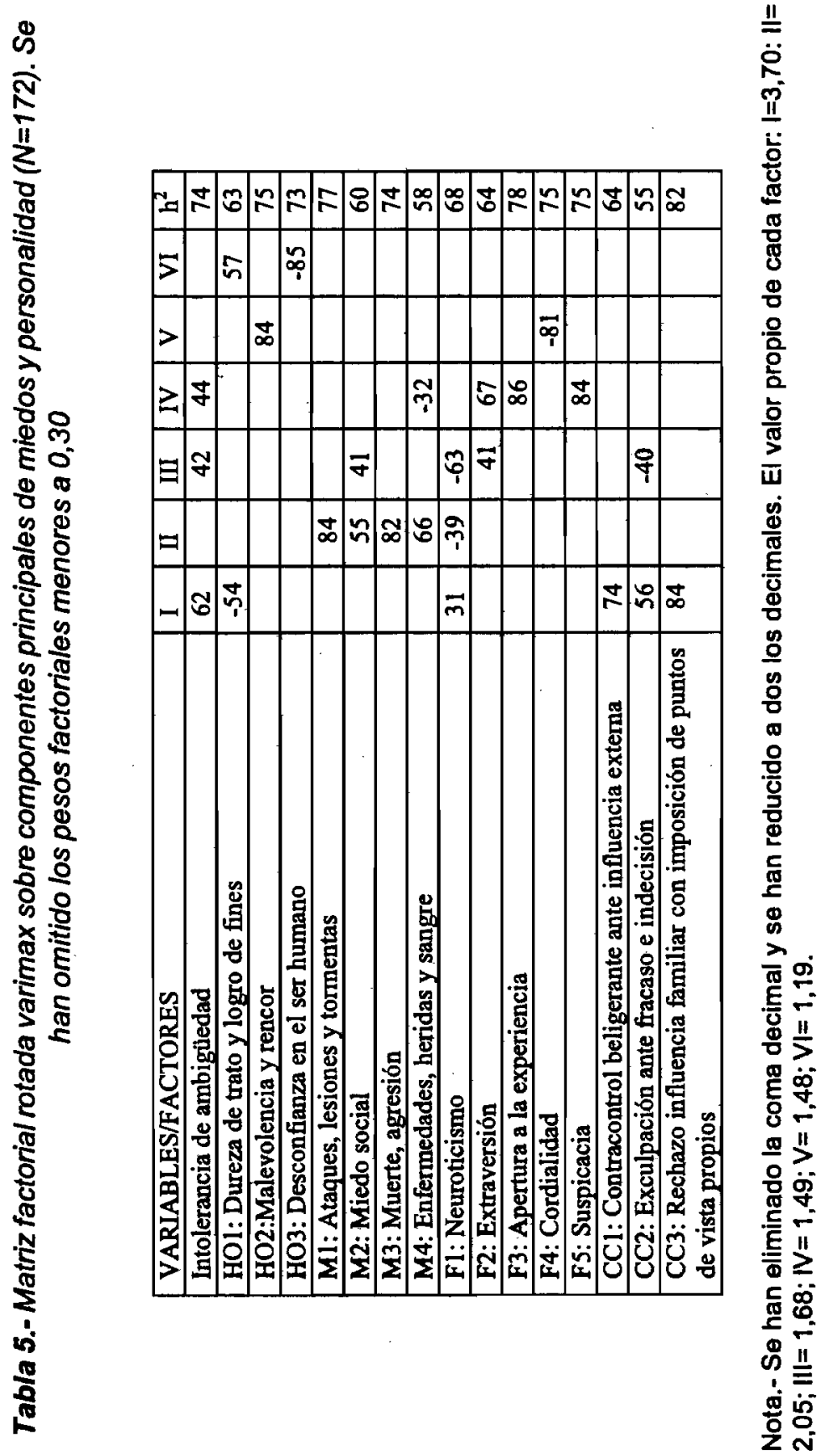


existe un peso no definitorio pero si de cierto interés de neuroticismo $(-0,39)$. Este resultado, que ya se había presentado en el análisis correlacional indicaría que el neuroticismo, tal y como es evaluado por el cuestionario de los cinco grandes no tendría mucho que ver con una vulnerabilidad a la ansiedad que podría derivarse de unos miedos altos en todos los factores.

El tercer factor posee un valor propio de 1,68 (10,52\% de varianza total explicada) y la mayor saturación $(-0,63)$ se da con el factor de neuroticismo. Acompañan para definir el factor, extraversión, intolerancia de la ambigliedad, miedo social y el rechazo de la exculpación ante el fracaso. Seria un factor de control personal (autocontrol), que no es independiente de extraversión y mostrarla a personas que controlan su vida, son un poco extravertidas, no toleran la ambigüedad y no exculpan a los demás ni a la sociedad por sus fracasos.

El cuarto factor tiene un valor propio de $1,48(9,24 \%$ de varianza total explicada) y se encuentra definido por tres de los cinco factores de los cinco grandes: apertura a la experiencia, suspicacia (que conlleva una acentuada responsabilidad en el desempeño de tareas) y extraversión. Además, aparecen indícios de intolerancia de la ambigüedad y falta de miedos de enfermedades, heridas y sangre. Serian marcadores de la persona trabajadora, cumplidora y responsable, abierta a los demás y a las nuevas experienclas y con facilidad para las relaciones personales, con cierta intolerancia de la ambigüedad.

El quinto factor posee un valor propio de 1,2 (un $7,44 \%$ de varianza total explicada) y parece claramente un factor de hostilidad manifiesta. Dos variables lo definen con claridad: la malevolencia y el rencor $(0,84)$ y el rechazo de cordialidad $(-0,81)$.

El sexto factor posee un valor propio de 1,05 (6.54\% de varianza total explicada) y parece factor complejo en el que a la vez se tiene una confianza en el ser humano y se defiende un trato duro con los demás.

El análisis factorial muestra con bastante claridad que los miedos formarían un sistema funcional que es bastante independiente de los factores de personalidad medidos en este estudio. Las relaciones, cuando aparecen, se dan con neuroticismo y en un sentido distinto al esperado. Por otro lado, los cinco grande son parecen ser factores independlentes entre sl y las relaciones que se dan entre ellos tienen 
sentido psicológico. Contracontrol e intolerancia de la ambigüedad forman un patrón común de relaciones, del mismo modo que cordialidad lleva consigo un rechazo de malevolencia y rencor y dos de los tres factores de hostilidad ofrecen una relación negativa.

\section{RESUMEN Y DISCUSIÓN}

Por lo que se refiere a los resultados, lo primero que hay que señalar es que el análisis factorial sobre 100 elementos de miedos comunes da lugar a una estructura factorial robusta de cuatro factores, con una consistencia interna satisfactoria y que hacen sentido psicológico. Estos factores se mantienen estables en un rango de edad de 17 a 65 años al menos, en ambos géneros. $Y$ en lo que se refiere a las puntuaciones en cada grupo de edad, los hombres tienden a presentar una puntuación mayor que las mujeres, aunque estas diferencias no son siempre estadisticamente significativas (en la mitad aproximadamente de las comparaciones tan solo $y$, además, concentradas en algún factor más que en otros. Por otro lado, el análisis factorial ha arrojado una estructura factorial distinta para cada género y las diferencias básicamente se sitúan en la relevancia y estructuración de los miedos sociales.

Un primer resultado que llama la atención por lo novedoso es el que se refiere a las mayores puntuaciones (a veces con diferencias estadisticamente significativas y otras veces no) que se han obtenido, en general, $y$ en los grupos de edad, de los hornbres respecto a las mujeres. En niños (Pelechano, 1981, 1984) con el mismo instrumento los resultados eran los opuestos. Una explicación posible de este fenómeno ya fue apuntada en aquellas publicaciones: por aprendizaje cultural, los hombres (entonces) estaban más presionados a no manifestar miedo (era considerado síntoma de debilidad) y ello formaría parte del estereotipo social según el cual el hombre debe expresar poco sus emociones y sentimientos, frente a lo deseable en el caso de las mujeres. No es que los hombres antes eran menos miedosos que las mujeres $y$ ahora no, sino que antes estaba menos permitido expresar emociones (entre ellas el miedo) en los hombres que en las mujeres. Uno de los efectos que ha podido traer consigo el movimiento de liberación de la mujer, y las reiteradas peticiones para que los 
hombres expresen sus sentimientos y emociones ha podido ayudar a una inversión del fenómeno y, en nuestros dias, el hombre es animado a expresar emociones y, a la vez, la mujer, o a esconderlas o a controlar su expresión. Este mismo fenómeno es el que explicarla los cambios en la estructura factorial de los miedos en hombres y en mujeres.

Un segundo tipo de reflexión se relaciona con las diferencias aducidas al principio entre miedo y ansiedad. En la medida en que neuroticismo, tal y como lo hemos evaluado nosotros, fuera un marcador de vulnerabilidad a la ansiedad, ésta se presenta no solamente como independiente de los factores de miedos sino con una relación negativa, lo que abundarla en la diferenciación entre miedos y ansiedad en un sentido similar al defendido por Barlow. Sin embargo, en la medida en que «neuroticismo" tal y como se ha evaluado aqui no tenga que ver directamente con ansiedad sino con un aspecto, procesual o dinámicamente muy anterior a la ansiedad clinica, se podría seguir manteniendo que los miedos generalizados se "transforman" en ansiedad y que en esa transformación, las escalas de neuroticismo que se encuentran más cerca de la sintomatologla y acepción clínica, ofrecerian un panorama distinto. Con los resultados actuales no se puede decidir sobre esta cuestión.

De todos los factores de personalidad, aparte nouroticismo, solamente se encuentran de ciertos indicios relacionales de los miedos con extraversión (miedo social) y, a la vez, la extraversión (menos) y más decididamente, la apertura a la experiencia y la suspicacia parecen buenos antídotos para el miedo a enfermedades, heridas y sangre. Los procesos por los cuales esto sucede distan mucho de estar claros, aunque resulta un aspecto prometedor de posibles aplicaciones en el tratamiento de fobias.

Algunas limitaciones serias tiene este trabajo: en primer lugar, que se trata de datos transversales y la presunción de tendencias simples y lineales con este tipo de datos es problemática, sin embargo, on ausencia de datos longitudinales y de varias generaciones, los actuales pueden apuntar a estudios más complejos en los que se confirmen estos resultados. En segundo lugar, el número de personas sobre el que se han llevado a cabo los estudios con personalidad no es muy grande, por lo que sería necesarlo contrastar los resultados que aqui se han presentado. En ausencia de estudios longitudinales al respecto 
$y$ de estudios sobre el tema en nuestro contexto cultural, han animado al autor a presentar los resultados a consideración del lector.

\section{REFERENCIAS BIBLIOGRÁFICAS}

APA (2007).- Dictionary of Psychology, Washington DC, American Psychological Association.

Avia, M.D., Sanz, J., \& Bernardos, M.L. (1997).- Versión española del inventario de personalidad NEO revisado NEO-PI-R de Paul $T$. Costa y Robert R. McCrae, Departamento de Psicología Clínica, Universidad Complutense de Madrid. Edición expenimental.

Barlow, D.H. (1988). - Anxiety and its disorders: The nature and treatment of anxiety and panic, New York, Guilford Press.

Costa, P.T. \& McCrae, R.R. (1992).- Revisited NEO personality inventory (NEO-PI-R) and NEO five-factor inventory (NEO-FFI) professional manual, Odessa, FL, Psychological Assessment Resources.

Gullone, E., King, N.J. \& Ollendick, T. H. (2000).- The development and psychometric evaluation of the Fear Experiences Questionnaire: an attempt to disentangle the fear and anxiety constructs, Clinical Psychology and Psychotherapy, 7, 61-75.

Pelechano, V. (1981).- Miedos infantiles y terapia familiar-natural, Valencia, Alfaplús.

Pelechano, V. (1984).- Programas de intervención psicológica en la infancia: miedos, Valencia, Alfaplús.

Pelechano, V. (2000).- Cuestionario de hostilidad y malevolencia (HOSTYMAL), Análisis y Modificación de Conducta, 26, 779-816.

Pelechano, V. y Aguilera, F.J.(2004).-El cuestionario IA de intolerancia de la ambigüedad de Pelechano para adultos: presentación y datos de validación interna, convergente y diferencial, Análisis y modifícación de Conducta,30, 627-662.

Pelechano, V., Peñate. W. y González, M. (1997).- Un cuestionario de contracontrol y datos de validez de constructo, convergente, diferencial y evolutiva, Análisis y Modificación de Conducta, 23, 309-354.

Sandin, B. (1997).- Ansiedad, miedos y fobias en niños y adolescentes, Madrid, Dickinson.

Valiente, R.M., Sandín, B. y Chorot, P. (2003).- Miedos en la infancia y la adolescencia, Madrid, UNED. 